\title{
Measurement of Static and Dynamic Magneto-Viscoelasticity in Facile Varying pH Synthesized $\mathrm{CoFe}_{2} \mathrm{O}_{4}$-Based Magnetic Fluid
}

\author{
Akash Mishra $^{1,2,3}$, Saurabh Pathak ${ }^{\circledR 1,4,5}$, Prashant Kumar ${ }^{1,5}$, Arjun Singh ${ }^{1,5}$, Komal Jain ${ }^{1,5}$, \\ Raghav Chaturvedi ${ }^{6}$, Dinesh Singh $^{1}$, G. A. Basheed ${ }^{1,5}$, and R. P. Pant ${ }^{1,5}$ \\ ${ }^{1}$ Indian Reference Materials Division, CSIR-National Physical Laboratory, New Delhi 110012, India \\ ${ }^{2}$ University Teaching Department, School of Nanotechnology, Rajiv Gandhi Proudyogiki Vishwavidyalaya, \\ Bhopal 462036, India \\ ${ }^{3}$ Indian Institute of Technology Bombay, Mumbai 400076, India \\ ${ }^{4}$ School of Engineering, RMIT University, Melbourne, VIC 3000, Australia \\ ${ }^{5}$ Academy of Scientific and Innovative Research, CSIR-National Physical Laboratory Campus, \\ New Delhi 110012, India \\ ${ }^{6}$ Department of Physics, Indian Institutes of Science Education and Research, Thiruvananthapuram 695551, India
}

\begin{abstract}
In this work, we have investigated the size induced on the viscoelasticity of $\mathrm{CoFe}_{2} \mathrm{O}_{4}$ magnetic nanofluids (MNFs). The focus of this work is to provide an insight into the effect of varying particle size on viscoelastic properties of $\mathrm{CoFe}_{2} \mathrm{O}_{4} \mathrm{MNFs}$ with varying $\mathrm{CoFe}_{2} \mathrm{O}_{4}$ nanoparticle sizes. Kerosene-based MNFs containing surfactant coated $\mathrm{CoFe}_{2} \mathrm{O}_{4}$ were synthesized using standard optimized coprecipitation method, and variations in average mean size $\sim 9-30 \mathrm{~nm}$ have been synthesized. The physical properties, such as structural and morphology, have been investigated to confirm the purity, and variation in size. Steady-state and oscillatory mode measurements were performed using a magneto-rheometer to investigate the field-induced magneto-rheology. The steady-state rheograms (viscosity vs. shear rate curve) has been well fitted with power-law $\eta=c \dot{\gamma}^{n}+\eta_{\infty}$, confirming the shear thinning behavior with $n \leq 1$. Furthermore, magneto-sweep rheograms (viscosity vs. magnetic field) has been used to investigate the steady increase in viscosity, which is due to the formation of a chain-like structure in the field direction causing an interruption in the smooth streamline flow of the MNFs. Furthermore, dynamic oscillatory measurement shows the transition between G' and G' with applied dynamic strain confirming solid-liquid phase transition behavior. Field-induced viscoelastic behavior in static and dynamic mode provides significant information for optimization of MNFs for various applications.
\end{abstract}

Index Terms - Cobalt ferrites, magnetic fluid, magneto-viscoelasticity, rheology, spinal ferrites.

\section{INTRODUCTION}

$\mathbf{V}$ ISCOELASTIC properties of magnetic nanofluids (MNFs) play an essential role in the device development, as magnetic control on fluid behavior is a promising field in numerous applications. MNFs commonly known as ferrofluids are synthetic fluids having ultra-fine ferromagnetic and ferrimagnetic particles in the size range $(2-40 \mathrm{~nm})$ dispersed in the magnetically passive medium. [1] These fluids show non-Newtonian behavior when subject to the external magnetic field. Magnetic nanoparticles (MNPs) dispersed in MNFs form a chain-like structure in the field direction, which causes hindrance to the streamline fluid flow, which leads to an increase in viscosity. The morphology, dilution, carrier fluid, and surfactant of MNFs are the key parameters which strongly influence the magneto-viscous effect (MVE). Optimization of MVE with varying particle size is essential for various device applications in engineering (motors, bearings, actuators, sensors, and thermal applications), as well as biological application (hyperthermia treatment, drug delivery) [2]-[5].

Rheological properties of MNFs were significantly affected by stability, density, particle size distribution, coercive field,

Manuscript received December 14, 2018; revised May 6, 2019 and August 1, 2019; accepted August 18, 2019. Date of current version November 18, 2019. Corresponding authors: S. Pathak and R. P. Pant (e-mail: saurabh.pathak28@gmail.com; rppant@nplindia.org).

Color versions of one or more of the figures in this article are available online at http://ieeexplore.ieee.org.

Digital Object Identifier 10.1109/TMAG.2019.2936802 and saturation magnetization. Other than these factors, the base fluids, surfactants, anticorrosion, and additives also play a vital role in the performance of MNFs. Earlier, many studies have been carried out by researchers investigating the MVE and relative microstructure in the colloidal suspension of superparamagnetic (SP) particles to maximize the performance of MNFs.

Liu et al. [6] studied the volume concentration of SP particles on magneto-induced stress and established a microstructural dependence for magneto-viscous (MV) properties. Also, particle-level-dynamic simulation has been used to reveal the formation of the transverse stretched net-like structures, which leads to enhanced transversal stress effect. The volume fraction of the SP particle size plays a vital role in the formation of a chain-like structure. At the optimized volume fraction, the axial and transverse stress enhancement is maximum. Noorjahan et al. [4] investigated the effect of dilution on dipolar interaction and magneto-viscoelasticity of magnetic fluids. The interparticle dipolar interaction plays a vital role in MVE of the MNFs system. Also, they have corroborated their steady-state and oscillatory results with different models, like power law (thinning behavior), Bingham model (yield stress), and De Gans Theory (dynamic behavior). Furthermore, they have also shown Mason number as a function of specific viscosity, which gives a perfect scaling with power-law exponent less than one, which confirms the shear thinning behavior. Paul et al. [7] reported an extensive study on the influence 
of different particle concentration loading of $\mathrm{Fe}_{3} \mathrm{O}_{4}$ particles dispersed in water-based MNFs. They have concluded that non-Newtonian behavior manifests more prominently with an increase in the magnetic field. Also, they have performed the steady-state rheological measurement and fitted experimental data with "Harshell and Bulkley" (H. B. Model) to calculate yield stress. A good agreement between experimental data and fitted data has been observed. Furthermore, they have concluded that the yield stress of MNFs has been improved significantly with increment in particle loading and magnetic field [6], [8]-[11].

The main focus of this work is to experimentally investigate and establish a correlation between rheological properties and varying size of MNPs. $\mathrm{CoFe}_{2} \mathrm{O}_{4}$ MNPs were synthesized by standard coprecipitation method, and three different MNF samples $\mathrm{A}, \mathrm{B}$, and $\mathrm{C}$ were prepared by precipitation at three different $\mathrm{pH}$ values 8,10 , and 12 , respectively. XRD and highresolution transmission electron microscope (HRTEM) have been used to investigate the purity and crystallinity of all the samples. Rheological measurements were done in both static and dynamic mode. Non-Newtonian shear thinning behavior of samples was confirmed by static rheological measurements, and dynamic oscillatory measurement confirms the solid-liquid phase transition. In this work, we have presented a detailed experimental study on rotational and oscillatory behavior. The experimental results are in good correlation with theoretical models showing the viscoelastic behavior of fluid samples.

\section{SYNTHESIS}

\section{A. Materials}

The raw materials used for the synthesis of cobalt ferrite nanocrystalline particles are iron (III), chloride anhydrous $\left(\mathrm{FeCl}_{3}\right)$, cobalt (II), chloride hexahydrate $\left(\mathrm{CoCl}_{2} \cdot 6 \mathrm{H}_{2} \mathrm{O}\right)$, sodium hydroxide $(\mathrm{NaOH})$, oleic acid pure $\left(\mathrm{C}_{18} \mathrm{H}_{34} \mathrm{O}_{2}\right)$, hydrochloric acid $(\mathrm{HCl})$, acetone, and kerosene, and were procured from Merck Chemicals. All the chemicals were used as received, without any further purification. Deionized water (DI) was obtained by SHRO-plus DI (18 M 2 ) system.

\section{B. Preparation}

For the synthesis of MNPs, we have adopted the chemical coprecipitation technique. We have taken a salt solution of $\mathrm{Co}^{2+}$ and $\mathrm{Fe}^{3+}$ ions in the ratio of 1:2. The salt solution was mixed and homogenized at $80{ }^{\circ} \mathrm{C}$ for $15 \mathrm{~min}$ at $500 \mathrm{rpm}$. Temperature is maintained constant, and $1 \mathrm{M} \mathrm{NaOH}$ solution is added dropwise to this mixture ( $\sim 9 \mathrm{ml} / 5 \mathrm{~min})$ to increase the $\mathrm{pH}$ of the solution. The change in color of the solution confirms the formation of a precipitate. Three samples A, $\mathrm{B}$, and $\mathrm{C}$ were prepared at three precipitating $\mathrm{pH} 8,10$, and 12 by adding different volumes of $\mathrm{NaOH}$ solution. The mixture was stirred under the same condition for one hour. The precipitate is then washed several times using magnetic decantation with water and ethanol to remove any trace of impurities. The precipitate is then coated with oleic acid by stirring for $20 \mathrm{~min}$. The coated particles are again filtered and washed with DI water and acetone to remove all the impurities. Following this, the obtained precipitate is dried for $18 \mathrm{~h}$ at $70{ }^{\circ} \mathrm{C}[12]-[14]$.

\section{CHARACTERIZATION}

\section{A. Structural and Morphological Characterization}

Structural (crystallinity and phase) and morphology (shape and size distribution) have been confirmed by XRD and HRTEM. The structural characterization of synthesized MNF was done using multipurpose XRD (Rigaku Ultima IV). The identity, phase, and crystallite size of the sample were determined by RIGAKU XRD at a slow scan speed of $0.5^{\circ} / \mathrm{min}$ in the range $20^{\circ}-80^{\circ}$. XRD curves of all samples were fitted with the Williamson-Hall (W-H) model to investigate crystallite size and strain. Surface morphology (shape and size) of the samples were investigated using HRTEM (FEI, Tecnai G2, F30, STWIN).

\section{B. Rheological Characterization}

Static and dynamic rheological measurements were performed using magneto-rheometer to investigate the viscoelastic behavior of MNFs. The effect of particle size distribution on MVE is observed by keeping all other parameters, such as particle concentration, carrier fluid, surfactant, etc., which can cause a change in MVE, the same. These measurements were corroborated with theoretical predictions, and results were fitted with various models to provide better insight into the viscoelasticity of MNFs. The viscoelastic properties of the samples were investigated using magneto-rheometer (Anton Paar GmbH MCR 301). The measurement was performed using standard rotation geometry MRD-70, on spindle PP-20 with a transverse magnetic field, which is coaxial to the direction of parallel plate geometry. Thermostatic bath $\left( \pm 0.1^{\circ} \mathrm{C}\right)$ was used to maintain the constant temperature, and the gap between the parallel plates was optimized to $1 \mathrm{~mm}$.

\section{RESUlTS AND Discussion}

\section{A. Characterization of Nanoparticles}

The XRD pattern of prepared $\mathrm{CoFe}_{2} \mathrm{O}_{4}$ nanoparticles of samples A, B, and C are shown in Fig. 1(a). The characteristic diffraction peaks perfectly match with the cubic crystalline structure of $\mathrm{CoFe}_{2} \mathrm{O}_{4}$ (ASTM card no.: 22-1086). The peak positions at $2 \theta=30.084,35.437,43.058,56.973$, and 62.585 perfectly match with planes 220, 311, 400, 511, and 440, respectively, of cubic spinel ferrite structure

$$
\beta_{\mathrm{hkl}} \cos \theta=\frac{k \lambda}{D}+4 \epsilon \sin \theta .
$$

Equation (1) is known as Williamson Hall equation, where $\beta_{\mathrm{hkl}}$ is full width at half maximum (FWHM) of different peaks, $\mathrm{k}$ is dimensionless shape factor, $\lambda$ is the wavelength of $\mathrm{x}$-ray, $D$ is crystallite size, and $\epsilon$ is the microstrain occurs from the peak broadening. In (1), the strain is assumed to be uniform in all crystallographic directions. In Fig. 1(b)-(d), $\beta \cos \theta$ is plotted with respect to $4 \sin \theta$ for all the peaks of $\mathrm{CoFe}_{2} \mathrm{O}_{4}$ for the three samples. Strain and particle size are calculated from the slope and $y$-intercept of the fitted line, are shown in Table I. From the lattice parameters calculations, it is observed that crystallite size has been reduced on increasing $\mathrm{pH}$ due to decreasing particle size, and strain gets increased due to lattice shrinkage. The reduction in crystallite size is observed, 

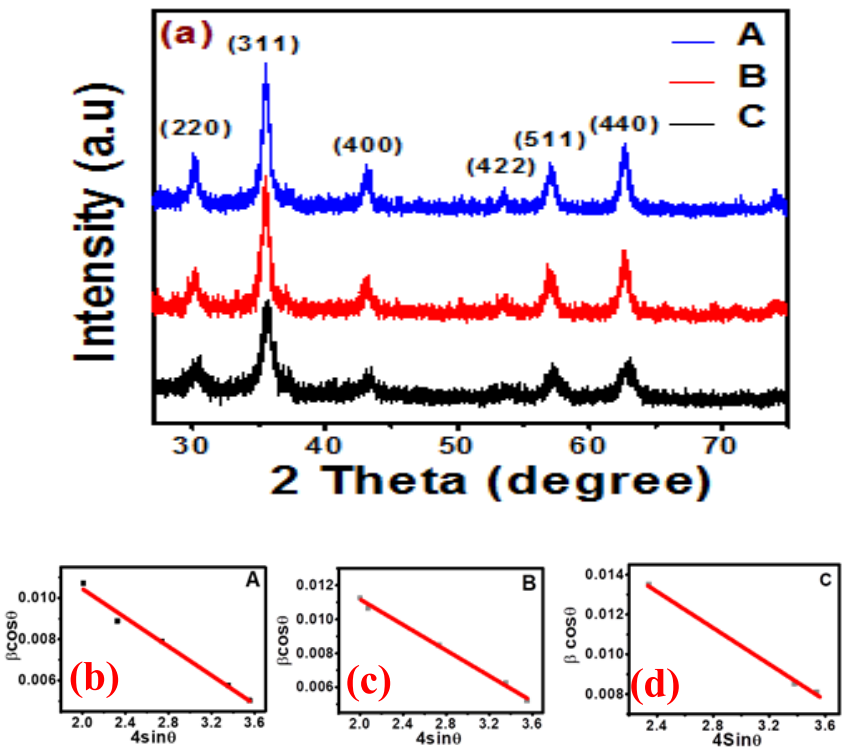

Fig. 1. (a) XRD pattern of $\mathrm{CoFe}_{2} \mathrm{O}_{4}$ of samples $\mathrm{A}, \mathrm{B}$, and $\mathrm{C}$. (b)-(d) W-H plot of samples A, B, and C, respectively. From W-H plot, the strain is calculated from the slope, and the crystallite size is obtained from the $y$-intercept of the fit.

TABLE I

Structural Parameters of $\mathrm{CoFe}_{2} \mathrm{O}_{4}$ at VARying $\mathrm{pH}$

\begin{tabular}{ccc}
\hline Sample & $\begin{array}{c}\text { Crystallite Size } \\
(\mathbf{n m})\end{array}$ & Strain (\%) \\
\hline Sample A & $8.8302(6)$ & $0.0035(6)$ \\
\hline Sample B & $8.235(1)$ & $0.003(5)$ \\
\hline Sample C & $6.390(4)$ & $0.004(1)$ \\
\hline
\end{tabular}

which is primarily due to the increased number of nucleation sites at higher $\mathrm{pH}$ due to which, particle size has also decreased.

\section{B. TEM Analysis}

For detailed investigation about microstructure, all three samples A, B, and C were characterized by HRTEM (FEI, Tecnai G2, F30, STWIN) operated at $300 \mathrm{keV}$ accelerating voltage. Fig. 2(a), (d), and (g) are the TEM images of samples $\mathrm{C}, \mathrm{B}$, and $\mathrm{A}$, respectively. These TEM images show a significant variation in the nanoparticle's sizes with a change in the precipitating $\mathrm{pH}$. In sample $\mathrm{C}$, the particles size is in the range from 2 to $15 \mathrm{~nm}$, whereas the particles size of sample $\mathrm{B}$ is in the range from 5 to $30 \mathrm{~nm}$, and in sample A, the size of particles is in the range from 5 to $40 \mathrm{~nm}$. So we can clearly see that the average particle size is decreasing with increase in precipitating $\mathrm{pH}$. Furthermore, Fig. 2(b), (e), and (h) are the selected area electron diffraction (SAED) pattern images of $\mathrm{CoFe}_{2} \mathrm{O}_{4}$ nanoparticles of $\mathrm{C}, \mathrm{B}$, and A, respectively, in the reciprocal space. Dotted rings in the SAED pattern confirm the polycrystalline nature of particles. SAED pattern in Fig. 2(b) of $\mathrm{CoFe}_{2} \mathrm{O}_{4}$ nanoparticles of sample $\mathrm{C}$ shows the interplanar spacing 2.97 and $2.53 \AA$ corresponding to [220] and [311] plane of $\mathrm{CoFe}_{2} \mathrm{O}_{4}$, respectively.
In Fig. 2(e) and (h), SAED pattern of $\mathrm{CoFe}_{2} \mathrm{O}_{4}$ nanoparticles of sample B and A clearly shows the interplanar spacing 4.85, 2.97, and $2.53 \AA$ corresponding to [111], [220] and [311] plane, respectively, of $\mathrm{CoFe}_{2} \mathrm{O}_{4}$ nanoparticles. These results are also in good agreement with XRD data of $\mathrm{CoFe}_{2} \mathrm{O}_{4}$ nanoparticles. Fig. 2(c), (f), and (i) are the high-resolution TEM images of $\mathrm{CoFe}_{2} \mathrm{O}_{4}$ nanoparticles of $\mathrm{C}, \mathrm{B}$, and $\mathrm{A}$, respectively. Interplanar spacing 2.53 and $2.1 \AA$, which corresponds to [311] and [400] atomic plane of $\mathrm{CoFe}_{2} \mathrm{O}_{4}$ nanoparticles of $\mathrm{C}$ in HRTEM image [Fig. 2(c)] and also the particle size is near to $9.9 \mathrm{~nm}$ in this image. Interplanar spacing 2.53 and $2.97 \AA$, which corresponds to [311] and [220] atomic plane of $\mathrm{CoFe}_{2} \mathrm{O}_{4}$ nanoparticles of $\mathrm{B}$ in HRTEM image [Fig. 2(f)] and particle size is near to $20 \mathrm{~nm}$ in HRTEM image. Interplaner spacing $2.53 \AA$ is corresponding to [311] atomic plane of $\mathrm{CoFe}_{2} \mathrm{O}_{4}$.

\section{Rheological Properties}

1) Effect of Steady Shear on Viscosity: The dependence of viscosity on shear rate has been studied under varying shear rate $\left(0-200 \mathrm{~s}^{-1}\right)$ at constant field strength $0.18 \mathrm{~T}$. The applied magnetic field shows a very strong influence on all the samples. The particle size of sample A is large as compared to sample B, followed by sample C. Fig. 3(a) shows the dependence of viscosity on the shear rate at different samples of $\mathrm{CoFe}_{2} \mathrm{O}_{4}$ MNF. The flow curve of all three samples can be divided into three regions;

1) At very low shear rate $\left(0-5 \mathrm{~s}^{-1}\right)$, particles form chain structures in the direction of the field and show high viscosity due to strong dipolar interaction. In this region, interparticle dipolar interaction is greater than in hydrodynamic forces.

2) At moderate shear rate $\left(5-20 \mathrm{~s}^{-1}\right)$, the chains start breaking due to greater applied forces, and thus, the dominance of hydrodynamic forces on magnetic forces. Thus, viscosity starts decreasing with an increase in shear rate. In this region, the fluid shows nonNewtonian shear thinning behavior.

3) At high shear rate (20-200 s $\left.\mathrm{s}^{-1}\right)$, chains get completely destroyed, and MVE diminishes. In this region, fluid displays Newtonian behavior. It can be noted from Fig. 3 that the viscosity of sample A is much greater as compared to viscosity of samples Band C. This is due to the fact that the dipolar interaction of particles at sample A is very high followed by samples B and C. Due to this, the chain formation of sample A is strong and less number of chains breaks in the shear rate range $\left(0-200 \mathrm{~s}^{-1}\right)$ as compared to other samples. The chain formation in the magnetic field at sample $\mathrm{C}$ is very weak, that breaks on a large scale and leading to the reduction in viscosity. Fig. 3(a). shows the viscosity versus shear rate curve that has been fitted with power law

$$
\eta=c \dot{\gamma}^{n}+\eta_{\infty}
$$

where $\mathrm{c}, \mathrm{n}$, and $\eta_{\infty}$ are fitting parameters, as shown in Table II. The value of exponent lies between -0.75 and -0.85 , which shows strong shear thinning behavior of all samples 


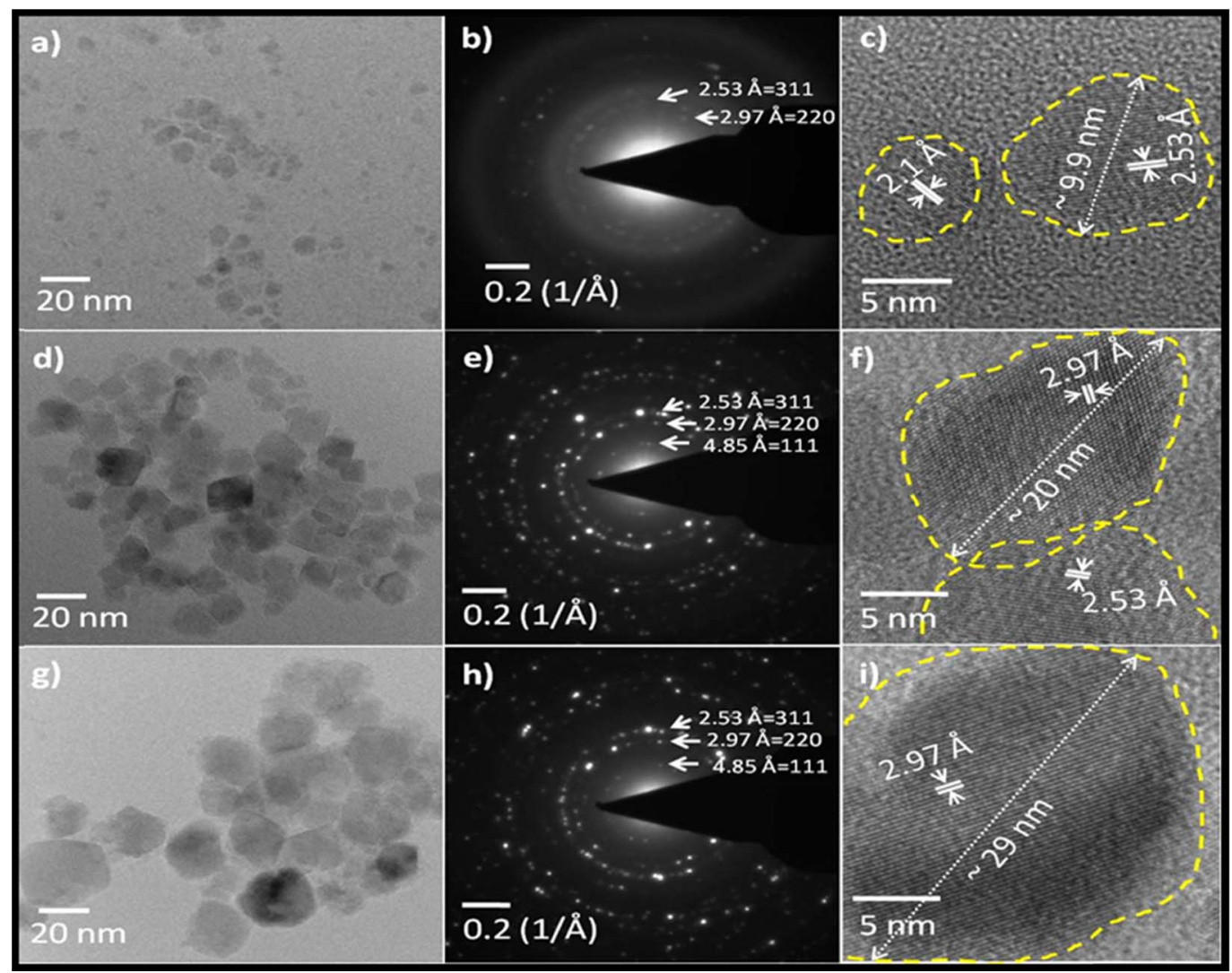

Fig. 2. (a), d), and g) are the TEM images of $\mathrm{CoFe}_{2} \mathrm{O}_{4}$ nanoparticles of $\mathrm{C}, \mathrm{B}$, and $\mathrm{A}$, respectively. Fig b), e), and h) are the $\mathrm{SAED}$ pattern of CoFe $2 \mathrm{O}_{4}$ nanoparticles of $\mathrm{C}, \mathrm{B}$, and A, respectively. Fig c), f), and i) are the HRTEM images of $\mathrm{CoFe}_{2} \mathrm{O}_{4}$ nanoparticles of C, B, and A, respectively.

TABLE II

VISCOELASTIC PARAMETERS FOR DIFFERENT SAMPLES

\begin{tabular}{cccccc}
\hline Sample & $\begin{array}{c}\boldsymbol{\tau}_{\boldsymbol{B}} \\
(\mathbf{P a})\end{array}$ & $\boldsymbol{\eta}_{\boldsymbol{P}}$ & $\boldsymbol{\Delta}$ & $\boldsymbol{\eta}_{\infty}$ & $\mathbf{n}$ \\
\hline $\mathbf{A}$ & $28(2)$ & $40.75(3)$ & $0.252(7)$ & $0.14(2)$ & $-0.845(2)$ \\
$\mathbf{B}$ & $7(1)$ & $17.15(9)$ & $0.295(6)$ & $0.116(2)$ & $-0.783(2)$ \\
$\mathbf{C}$ & $2.4(6)$ & $12.77(5)$ & $0.325(5)$ & $0.076(2)$ & $-0.755(7)$
\end{tabular}

of $\mathrm{CoFe}_{2} \mathrm{O}_{4}$. From Fig. 3 inset, it can be concluded that value $\eta_{\infty}$ decreases with a decrease in particle size, and $n$ increase with particle size decrease. Fig. 3(b) shows shear stress versus shear rate curve. At very low shear, there is a rapid increment in shear stress. This kind of behavior is well represented by Bingham law

$$
\tau=\tau_{\mathrm{B}}+\eta_{\mathrm{P}} \dot{\gamma}
$$

where $\tau$ is shear stress, $\tau_{\mathrm{B}}$ is yield stress, $\eta_{\mathrm{P}}$ is field dependent viscosity, and $\dot{\gamma}$ is the shear rate. Yield stress is the minimum value of shear stress at which chains start to break.

Fig. 3(b) shows the variation of yield stress with particles size of the sample. It is observed that yield stress decreases sharply on decreasing size. This is because, at small particle size, particles form small aggregates due to lower dipolar interaction and show paramagnetic behavior with a decrease in particle size, as grain boundaries increase. These grain boundaries have a larger surface area, due to which, the friction force between the two boundaries is very large in comparison to particles with lower grain boundaries. Thus, sample A displays lower viscosity in the absence of a magnetic field and higher viscosity in the presence of the field. During the rheology experiment, the samples are subjected to preshear of $20 \mathrm{~s}^{-1}$ for $30 \mathrm{~s}$. Following this, the constant magnetic field was applied, and the shear rate was ramped between 0 and $200 \mathrm{~s}^{-1}$. It should be noted that all the three samples showing similar behavior with varying stress value, which means varying shear rate on viscosity at the constant magnetic field is same for all three samples, but the value of viscosity changes. This is due to the different particle sizes of the three samples A, B, and C [15]-[17].

2) Magneto Sweep on Constant Shear Rate: The effect of varying magnetic field on the viscosity of all the samples at a constant shear rate $\left(50 \mathrm{~s}^{-1}\right)$ is shown in Fig. 4. All the three samples are subject to the varying fields between 0 and $1 \mathrm{~T}$ at a constant shear rate of $50 \mathrm{~s}^{-1}$. We have done the measurement in different shear rates, such as $10,25,50$, 100 , and $200 \mathrm{~s}^{-1}$. The response of all the samples are almost simple and showing the same trend, and the only variation is in magnitude. Thus, we have shown only $50 \mathrm{~s}^{-1}$ shear rate result. The increase in viscosity of all three samples on increasing magnetic field can be understood as on increasing field the magnetic flux density increases. This attributes to the formation of a chain-like structure, which increases the 

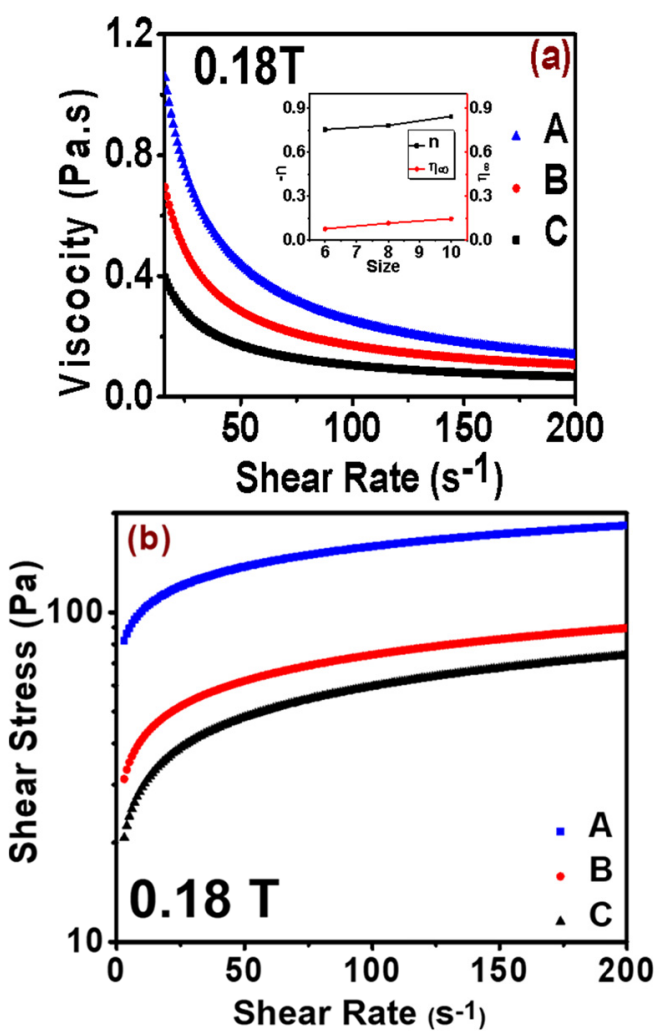

Fig. 3. (a) Effect of varying shear rate on viscosity at a constant field of $0.18 \mathrm{~T}$ for $\mathrm{CoFe}_{2} \mathrm{O}_{4}$ of samples A, B, and C. (b) Shear stress versus shear rate curve for different size particle samples $\mathrm{A}, \mathrm{B}$, and $\mathrm{C}$.

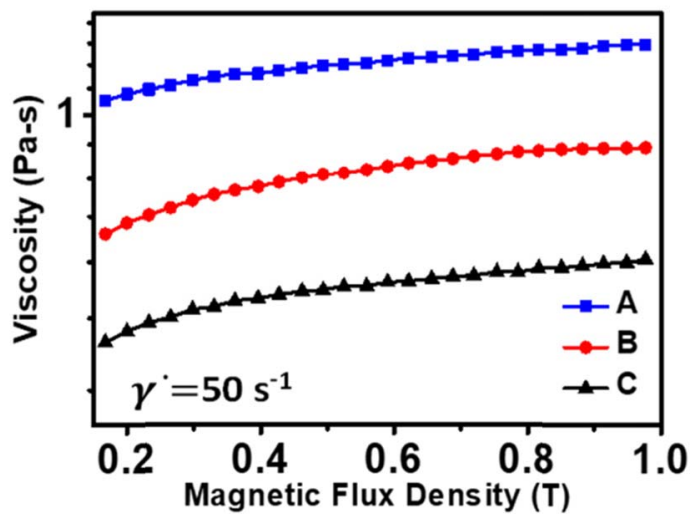

Fig. 4. Variation of MVE with different particle size samples A, B, and C; i.e., viscosity versus magnetic flux density at a constant shear rate of $50 \mathrm{~s}^{-1}$. It is evident from the figure that MVE is more prominent sample A.

resistance to fluid flow. However, the particle interaction of sample A is very strong. This leads to stronger dipolar interaction than hydrodynamic forces, resulting in impregnable structure and higher viscosity of sample A as compared to sample B and sample $\mathrm{C}$. The particle interaction of sample $\mathrm{C}$ is very weak, due to low resistance to fluid flow [18]. Due to this, the viscosity of sample $\mathrm{C}$ is lowest in comparison to samples A and B. Thus, we can conclude that with increase in particle size the MVE is more prominent and for lower size particles, dipolar interaction decreases which lead to lower viscosity of the sample and lower MVE [16], [19]-[21].
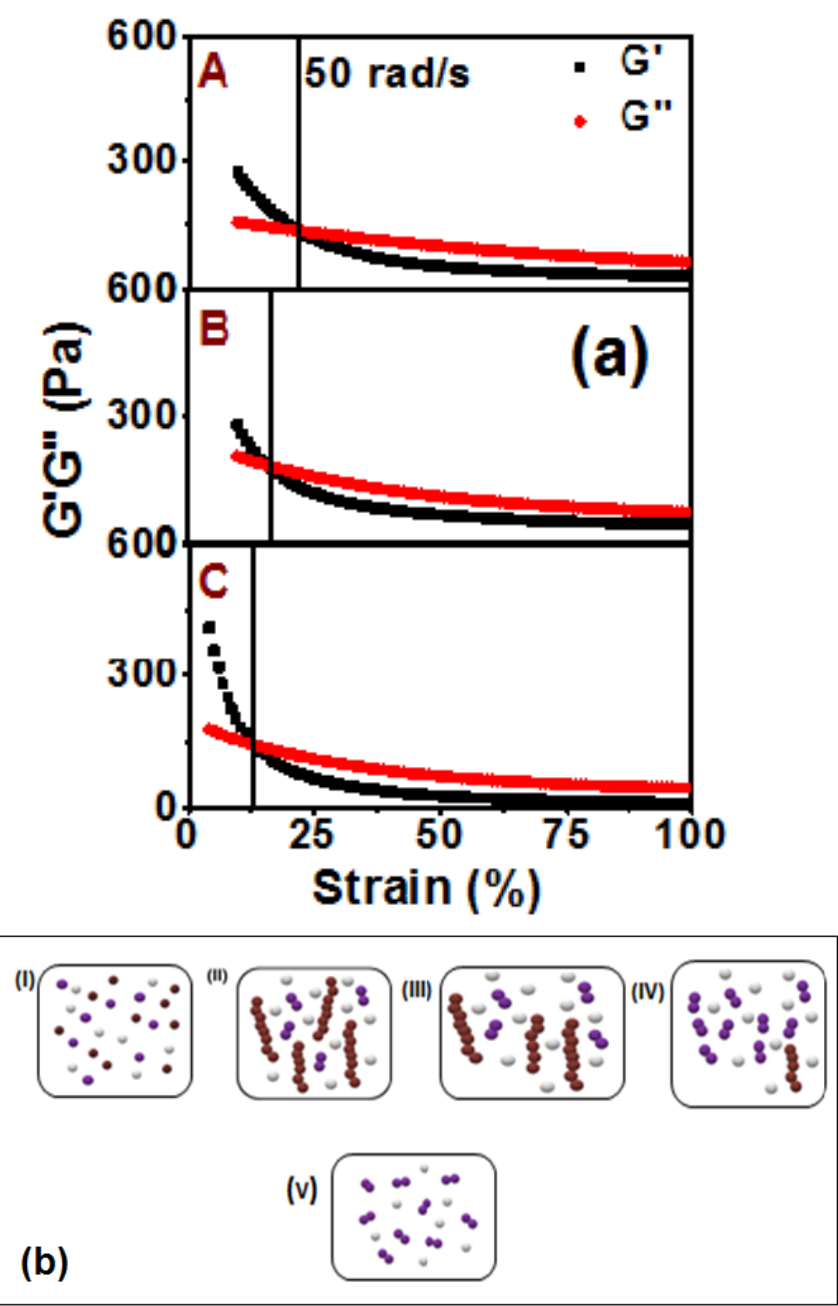

Fig. 5. (a) Strain Amplitude Sweep at a constant angular frequency and constant field. (b) Schema of application of strain. (I) in static equilibrium, (II) on applying magnetic field, (III) at crossover strain, (IV) on increasing strain, (V) at very high strain.

3) Strain-Amplitude Sweep: Strain amplitude sweep was performed under oscillatory shear to measure the viscoelastic properties of the sample. Fig. 5 shows strain amplitude sweep for all the three samples $\mathrm{A}, \mathrm{B}$, and $\mathrm{C}$ by varying strain from $0 \%$ to $100 \%$ at the constant magnetic field $(0.5 \mathrm{~T})$ and constant angular frequency (50 rad/sec). The storage/ elastic modulus (G') and loss/ viscous modulus (G') are plotted as a function of strain in this curve. At very low strain amplitude, the viscoelastic modulus is independent of strain. This region is called linear viscoelastic region (LVE). In this region $G^{\prime}>\mathrm{G}$,' the elastic behavior dominates the viscous behavior. This is due to dominant magnetostatic forces $\left(\mathrm{f}_{m}\right)$ over hydrodynamic forces $\left(\mathrm{f}_{h}\right)$, which confirms the existence of an elastic nature in the sample. With the increase in strain, the hydrodynamic forces start to dominate over magnetostatic forces and dissipation of stored energy increases.

Thus, the field-induced structure breaks down and displays liquid-like behavior. The crossover between G' and G', indicates the transition from solid to liquid-like behavior. For the three samples, the crossover of G' and G' at critical 
strain $\left(\gamma_{c}\right)$ is different. The critical strain of sample $\mathrm{A}$ is greater than sample $\mathrm{B}$, followed by sample $\mathrm{C}$.

This shows that the particles of sample A have greater storing capacity compared to other samples in the field. In sample $\mathrm{C}$, due to weak structuration, the value of critical strain is very low. Fig. 5(b) shows the effect of increasing strain on G' and G" of all the three samples. Solid circles represent the majority of elastic modulus G'. Purple and white circles represent short-chain and nonmagnetic carrier fluid molecules, respectively, which contribute to G." In static equilibrium, the MNF behaves like Newtonian fluid, consisting of all the magnetic particles and carrier fluid molecules freely, as shown in (I). On the application of the magnetic field, the chain framework structure holds all the magnetic particles and kerosene tightly containing all the longer chains, shorter chains, and free molecules of carrier fluid. So that the MNF behave like gel as shown in (II). At very small strain $\gamma<\gamma_{c}$, the chain framework withstands the small shear stress due to small strain. Thus, the chain framework is the same as the static equilibrium and G'> G." In (III), when $\gamma=\gamma_{c}$, the sum of long chains breaks into short chains, leading to an increase in G" and decrease in G'. Thus, at crossover strain $\mathrm{G}^{\prime}=\mathrm{G}$." In (IV), $\gamma>\gamma_{c}$, long chains destroyed significantly, and short chains and free particles increased, resulting in a further decrease in G' and increase in G." In (V), at very high strain, a complete destruction of long chains and fine dispersion of magnetic particles is reached, and viscoelastic liquid is obtained [22]-[24].

4) Frequency Sweep Measurement: Frequency sweep has been performed to measure the variation of storage modulus $\left(G^{\prime}\right)$ and loss modulus $\left(G^{\prime \prime}\right)$ with a varying angular frequency. Fig. 6. Shows frequency sweep for all the three samples by varying angular frequency between 0 and $100 \mathrm{rad} / \mathrm{sec}$, at constant strain (5\%) and constant field (0.5 T). The curve shows the effect on G' and G' under varying angular frequency. In this curve, the storage modulus ( $\left.\mathrm{G}^{\prime}\right)$ increases with an increase in angular frequency $(\omega)$, due to the formation of long chains.

On increasing frequency under the constant field, the length of chain increases, leading to an increase in the storing capacity of all the samples. From Fig. 6 it can be concluded that loss modulus of all three samples is nearly similar, but the storage modulus of sample A has larger slope compared to samples B and C. The particle size of sample A is large, which leads to faster and strong chain formation than other samples. Sample C has the lowest storage modulus slope due to the formation of small chains. It is observed that the loss modulus does not have much effect on the angular frequency, and it shows a constant linear behavior [7], [24]-[27].

5) Field-Induced Relaxation Study: The variation of viscosity of all the samples with time when subjected to the pulse of the magnetic field $(0.35 \mathrm{~T})$ is shown in Fig. 7 . In this figure, three regions can be seen as: 1) magnetic field OFF; 2) magnetic field ON; and 3) magnetic field OFF.

At the boundary of 1) and 2), there is an instant increase in viscosity due to enhanced dipolar interaction. On the removal of the magnetic field, again it shows the instant decrease in viscosity, due to dominating shear forces, which destroys the

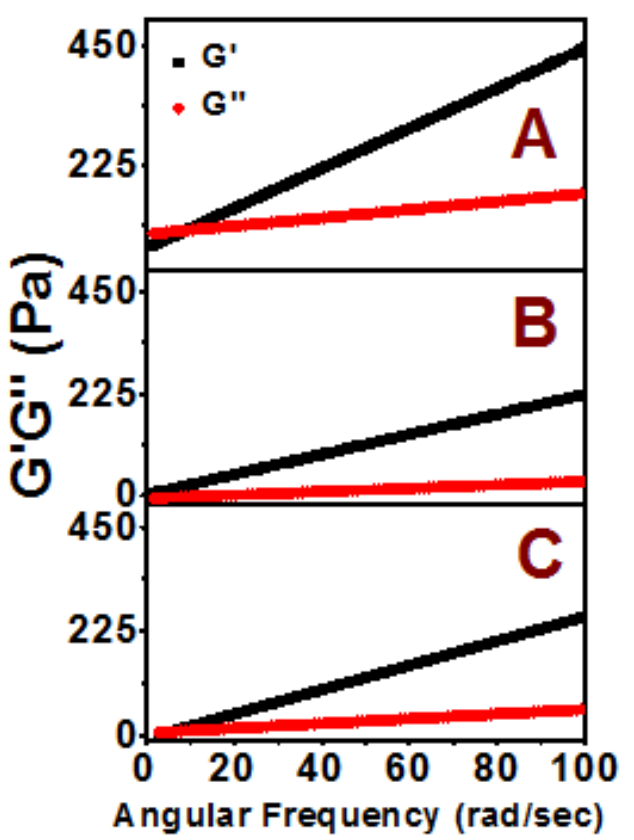

Fig. 6. Frequency sweep measurement at a constant strain and constant field.

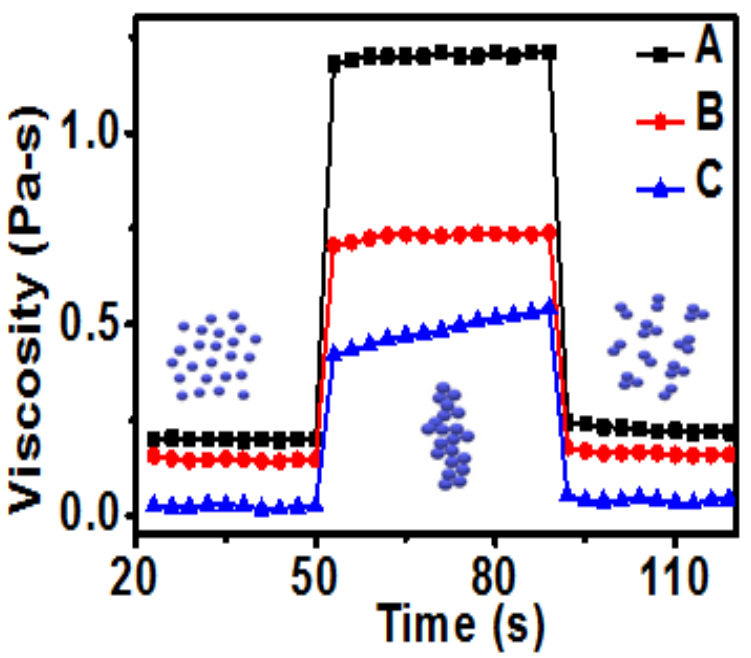

Fig. 7. Relaxation curve of $\mathrm{CoFe}_{2} \mathrm{O}_{4} \mathrm{MNF}$ of samples $\mathrm{A}, \mathrm{B}$, and $\mathrm{C}$ with schema, at a constant shear rate of $100 \mathrm{~s}^{-1}$.

magnetically induced chainlike structures. However, the viscosity in the region 3 ) is greater than the initial viscosity in the region 1). This indicates that part of the structure formed has not been destroyed completely or new aggregates have been reestablished. The decrease in viscosity with time in region 3) is related to gradual distortion of aggregate structures; i.e., breaking of chain-like structure, as shown in the schema of Fig. 7, which indicates that magnetic particles retain some memory of the applied magnetic field [28], and a very fast response time was observed for typical magneto rheological (MR) fluid [29]-[31].

\section{CONCLUSION}

In summary, the size-induced effect on static and dynamic viscoelastic properties of $\mathrm{CoFe}_{2} \mathrm{O}_{4}$ was investigated. 
$\mathrm{CoFe}_{2} \mathrm{O}_{4} \quad \mathrm{MNF}$ synthesized by chemical coprecipitation method with a size variation in the range $9-30 \mathrm{~nm}$ is achieved by varying the precipitating $\mathrm{pH}$. This has been confirmed with TEM micrograph and reduction in size with an increase in $\mathrm{pH}$ is evident from the micrograph. Furthermore, structural analysis was performed using XRD, which confirms the cubic spinel structure, and crystallite size and strain have been calculated using the $\mathrm{W}-\mathrm{H}$ method. With an increase in $\mathrm{pH}$, the crystallite size decreases, and strain in the particle increases. Static rheological measurements with varying magnetic field and shear rate were performed to investigate the MVE, which becomes more prominent with an increase in particle size. These results are corroborated with power law and Bingham model confirming non-Newtonian shear thinning behavior. Also, MNFs show a complete reversible transition with/without magnetic field condition as, in the absence of magnetic field, MNFs with lower particle size distribution shows higher viscosity, whereas in the presence of field it shows higher viscosity. The rheological measurements show non-Newtonian shear thinning behavior of MNF. The main reason for this transition can be predicted as the presence of higher grain boundaries in the absence of magnetic field which leads to increase in interparticle friction contributing higher viscosity, whereas in the presence of field interparticle dipolar interactions are greater leading to higher viscosity. Furthermore, the dynamic mode oscillation of strain amplitude and frequency sweep confirms the crossover transition from solid-liquid behavior. The transition strain amplitude shifts toward higher strain value with increase in particle size which confirms the higher MVE with an increase in size. Thus, we can conclude that the optimized MVE by controlling the size can be effective for various applications in numerous areas of engineering and biomedical.

\section{REFERENCES}

[1] N. Jahan, S. Pathak, K. Jain, and R. P. Pant, "Enchancment in viscoelastic properties of flake-shaped iron based magnetorheological fluid using ferrofluid," Colloids Surf. A, Physicochem. Eng. Aspects, vol. 529, pp. 88-94, Sep. 2017.

[2] K. Jain, S. Pathak, and R. P. Pant, "Enhanced magnetic properties in ordered oriented ferrofibres," RSC Adv., vol. 6, pp. 70943-70946, Jul. 2016.

[3] V. Kumar, A. Rana, M. S. Yadav, and R. P. Pant, "Size-induced effect on nano-crystalline $\mathrm{CoFe}_{2} \mathrm{O}_{4}$," J. Magn. Magn. Mater., vol. 320, pp. 1729-1734, Jun. 2008.

[4] G. A. B. Noorjahan, K. Jain, S. Pathak, and R. P. Pant, "Dipolar interaction and magneto-viscoelasticity in nanomagnetic fluid," J. Nanosci. Nanotechnol., vol. 18, pp. 2746-2751, Apr. 2018.

[5] S. Pathak, K. Jain, G. A. B. Noorjahan, V. Kumar, and R. P. Pant, "Magnetic fluid based high precision temperature sensor," IEEE Sensors J., vol. 17, no. 9, pp. 2670-2675, May 2017.

[6] T. Liu, X. Gong, Y. Xu, and S. Xuan, "Magneto-induced stress enhancing effect in a colloidal suspension of paramagnetic and superparamagnetic particles dispersed in a ferrofluid medium," Soft Matter, vol. 10, pp. 813-818, Nov. 2014.

[7] G. Paul, P. K. Das, and I. Manna, "Synthesis, characterization and studies on magneto-viscous properties of magnetite dispersed water based nanofluids," J. Magn. Magn. Mater, vol. 404, pp. 29-39, Apr. 2016.

[8] L. J. Felicia and J. Philip, "Effect of hydrophilic silica nanoparticles on the magnetorheological properties of ferrofluids: A study using optomagnetorheometer," Langmuir, vol. 31, pp. 3343-3353, Mar. 2015.

[9] A. Józefczak, T. Hornowski, Z. Rozynek, A. Skumiel, and J. O. Fossum, "Rheological study of dextran-modified magnetite nanoparticle water suspension," Int. J. Thermophys., vol. 34, pp. 609-619, Apr. 2013.
[10] J. Nowak, F. Wiekhorst, L. Trahms, and S. Odenbach, "The influence of hydrodynamic diameter and core composition on the magnetoviscous effect of biocompatible ferrofluids," J. Phys., Condens. Matter, vol. 26, no. 17, 2014, Art. no. 176004

[11] K. Shahrivar, A. L. Ortiz, and J. de Vicente, "A comparative study of the tribological performance of ferrofluids and magnetorheological fluids within steel-steel point contacts," Tribol. Int., vol. 78, pp. 125-133, Oct. 2014.

[12] K. Jain, S. Pathak, P. Kumar, A. Singh, and R. P. Pant, "Dynamic magneto-optical inversion in magnetic fluid using NanoMOKE," J. Magn. Magn. Mater, vol. 475, pp. 782-786, Apr. 2018.

[13] G. A. B. Noorjahan, S. Pathak, K. Jain, and R. P. Pant, "Improved magneto-viscoelasticity of cross-linked PVA hydrogels using magnetic nanoparticles," Colloids Surf. A, Physicochem. Eng. Aspects, vol. 539, pp. 273-279, Feb. 2018.

[14] S. Pathak, K. Jain, P. Kumar, X. Wang, and R. P. Pant, "Improved thermal performance of annular fin-shell tube storage system using magnetic fluid," Appl. Energy, vol. 239, pp. 1524-1535, Apr. 2019.

[15] M. T. López-López, A. Gómez-Ramírez, L. Rodríguez-Arco, J. D. G. Durán, L. Iskakova, and A. Zubarev, "Colloids on the frontier of ferrofluids. Rheological properties," Langmuir, vol. 28, pp. 6232-6245, Mar. 2012.

[16] J. Nowak, D. Wolf, and S. Odenbach, "A rheological and microscopical characterization of biocompatible ferrofluids," J. Magn. Magn. Mater. vol. 354, pp. 98-104, Mar. 2014.

[17] J. Wu, L. Pei, S. Xuan, Q. Yan, and X. Gong, "Particle size dependent rheological property in magnetic fluid," J. Magn. Magn. Mater, vol. 408 , pp. 18-25, Jun. 2016.

[18] M. H. Kim, K. Choi, J. D. Nam, and H. J. Choi, "Enhanced magnetorheological response of magnetic chromium dioxide nanoparticle added carbonyl iron suspension," Smart Mater. Struct., vol. 26, Aug. 2017, Art. no. 095006.

[19] R. Haghgooie and P. S. Doyle, "Transition from two-dimensional to three-dimensional behavior in the self-assembly of magnetorheological fluids confined in thin slits," Phys. Rev. E, Stat. Phys. Plasmas Fluids Relat. Interdiscip. Top., vol. 75, Jun. 2007, Art. no. 061406.

[20] H. Shahnazian and S. Odenbach, "Rheological investigations of ferrofluids with a shear stress controlled rheometer," J. Phys., Condens. Matter, vol. 20 , no. 20, 2008, Art. no. 204137.

[21] J. A. Ruiz-López, J. C. Fernández-Toledano, R. Hidalgo-Alvarez, and J. de Vicente, "Testing the mean magnetization approximation, dimensionless and scaling numbers in magnetorheology," Soft Matter, vol. 12, pp. 1468-1476, Nov. 2016.

[22] Y. Yang, L. Li, G. Chen, and W. Li, "Magnetorheological properties of aqueous ferrofluids," Nihon Reoroji Gakkaishi, vol. 34, no. 1, pp. 25-31, 2006.

[23] M. J. Pastoriza-Gallego, M. Pérez-Rodríguez, C. Gracia-Fernández, and M. M. Piñeiro, "Study of viscoelastic properties of magnetic nanofluids: An insight into their internal structure," Soft Matter, vol. 9 , pp. 11690-11698, Oct. 2013.

[24] S. Odenbach, "Microstructure and rheology of magnetic hybrid materials," Arch. Appl. Mech., vol. 86, pp. 269-279, Jan. 2016.

[25] S. E. Premalatha, R. Chokkalingam, and M. Mahendran, "Magneto mechanical properties of iron based MR fluids," Amer. J. Polym. Sci., vol. 2 , no. 4, pp. 50-55, 2012.

[26] J. M. Ginder, L. C. Davis, and L. D. Elie, "Rheology of magnetorheological fluids: Models and measurements," Int. J. Mod. Phys. B, vol. 10, pp. 3293-3303, Oct. 1996.

[27] M. Hassan, A. Zeeshan, A. Majeed, and R. Ellahi, "Particle shape effects on ferrofuids flow and heat transfer under influence of low oscillating magnetic field," J. Magn. Magn. Mater., vol. 443, pp. 36-44, Dec. 2017.

[28] K. Zhang, S. H. Piao, and H. J. Choi, "Hollow structured magnetic particles of $\mathrm{CoFe}_{2} \mathrm{O}_{4}$ and their magnetorheological characteristics," IEEE Trans. Magn., vol. 51, no. 11, Nov. 2015, Art. no. 2005904.

[29] F. Cousin, V. Cabuil, and P. Levitz, "Magnetic colloidal particles as probes for the determination of the structure of Laponite suspensions," Langmuir, vol. 18, pp. 1466-1473, Feb. 2002.

[30] B. Yendeti, G. Thirupathi, A. Vudaygiri, and R. Singh, "Field-dependent anisotropic microrheological and microstructural properties of dilute ferrofluids," Eur. Phys. J. E, vol. 37, p. 70, Aug. 2014.

[31] Y. D. Liu, J. Lee, S. B. Choi, and H. J. Choi, "Silica-coated carbonyl iron microsphere based magnetorheological fluid and its damping force characteristics," Smart Mater. Struct., vol. 22, no. 6, May 2013, Art. no. 065022. 